\title{
Do binding deficits account for age-related decline in visual working memory?
}

\author{
James R. Brockmole, Mario A. Parra, Sergio Della Sala, and Robert H. Logie \\ University of Edinburgh, Edinburgh, Scotland
}

\begin{abstract}
Remembering visual material, such as objects, faces, and spatial locations, over a short period of time (seconds) becomes more difficult as we age. We investigated whether these deficits could be explained by a simple reduction in visual working memory capacity or by an impairment in one's ability to form or maintain appropriate associations among pieces of related information. In three experiments, we used recognition and recall tests to address the efficacy with which older adults can create bound object representations by varying the number of features of each object that had to be remembered for a subsequent memory test. Results demonstrated that whereas older adults exhibited reduced memory capacity as compared with that of younger adults, both groups stored integrated object representations in visual working memory. These results are contrasted with other work that suggests that age-related memory decline is due, at least in part, to associative deficits.
\end{abstract}

Across a wide range of tasks, episodic memory abilities decline with age. In tests of long-term memory, these deficiencies have been linked, at least in part, to an increased difficulty in creating or retrieving the associations, or bindings, between single units of information. These units can range from individual objects down to the individual features that compose those objects. For example, older adults have more difficulty remembering word-word and face-name pairings despite preserved memory for the individual items (NavehBenjamin, 2000; Naveh-Benjamin, Guez, Kilb, \& Reedy, 2004), and, despite preserved memory for the colors and shapes presented in a visual array, older adults have more difficulty remembering the associations among each object's color, shape, and location (Chalfonte \& Johnson, 1996).

Here we extend previous discussions of these associative deficits in long-term memory to include visual working memory (VWM). One's ability to remember visual material, such as objects and faces, over a short period of time declines with age (see Reuter-Lorenz \& Sylvester, 2005). To what extent can this decline be accounted for by associative deficits? Objects are defined by different combinations of perceptual features, such as color, luminance, shape, size, orientation, texture, and so on. Hence, one's ability to recall a set of objects accurately depends on his or her ability to keep track of features that are associated with each other.

Studies with young (university-age) adults have shown that VWM performance is limited not by the number of features in a visual display, but by the number of objects that are present, suggesting that object features are bound and stored as singular structures in VWM (Allen, Baddeley, \& Hitch, 2006; Gajewski \& Brockmole, 2006; Johnson, Hollingworth, \& Luck, 2008; Luck \& Vogel, 1997; Vogel, Woodman, \& Luck, 2001; but see Wheeler \& Treisman, 2002).
In this article, we ask whether age is correlated with the qualitative changes in the representational format of VWM that reduce one's ability to combine features into individual object representations. If, as an associative deficit hypothesis would predict, older observers are forced to represent features individually, rather than as "chunks" of larger object representations, reduced performance on memorybased tasks should result. Some recent evidence favoring a binding deficit in VWM has indicated that older adults have increased difficulty maintaining associations between objects and their locations in VWM (Cowan, Naveh-Benjamin, Kilb, \& Saults, 2006; Mitchell, Johnson, Raye, Mather, \& D'Esposito, 2000). What is unclear is whether age-related VWM binding deficits extend to include the representation of the multiple surface features that define objects, such as color and shape - that is, whether such cognitive changes with age are specific to the long-term memory system, or if they originate in VWM. We tested this possibility in three experiments, each of which employed a different methodology that had been used in past research with young adults to test memory for bindings in VWM. This allowed us to assess older adults' binding abilities across a variety of display designs, retention intervals, and tasks.

\section{EXPERIMENT 1}

In Experiment 1, we used a change detection (i.e., recognition memory) task to investigate whether older adults' VWM capacity is limited by the number of objects or by the number of features that make up those objects. Following Luck and Vogel's (1997) seminal study on binding in VWM, we compared observers' memory for simple features to that for conjunctions of features. Participants judged whether

J. R. Brockmole, james.brockmole@ed.ac.uk 
temporally separated memory and test arrays of two to six colored shapes were the same or different. In the color block, only a color change could occur. In the shape block, only a shape change could occur. On any particular trial in the conjunction block, a change could occur to either an object's shape or its color. For each set size in this final conjunction condition, participants were required to remember twice as many features as in the single-feature blocks. If the object is the unit of VWM capacity, then performance when both features have to be remembered should be similar to that when only one feature has to be retained. Conversely, if observers have difficulty binding features into integrated object representations, then memory for visual displays should decline as the number of relevant features increases, even when the number of objects is held constant.

\section{Method}

Participants. Twelve healthy younger (mean age $=21.9$ years) and older (mean age $=70.7$ years) adults served as paid participants. Although, on average, the younger adults had slightly more education, the groups were matched in terms of verbal IQ as estimated from the Wechsler Test of Adult Reading (see Table 1). In this and in our subsequent experiments, all participants were screened for normal visual acuity and color vision, and older adults were screened for a history of abnormal neurological or psychiatric symptoms.

Stimuli. Stimuli consisted of arrays of two, four, or six objects placed randomly within an imaginary $3 \times 3$ square grid on a CRT monitor. Each object was created by randomly combining six colors and six shapes without replacement from a set of eight colors and shapes. On average, the objects subtended $1.8 \mathrm{~cm}$ horizontally and vertically. The grid subtended $6 \mathrm{~cm}$ horizontally and vertically. Viewing distance was not constrained.

Design and Procedure. The experimental procedure is illustrated in Figure 1. To suppress verbal rehearsal, each trial began with the presentation of two digits that the observer vocally repeated throughout the trial. The participant pressed a button to terminate the digits' display, which was then replaced with a fixation cross centered on a gray background. A second buttonpress initiated the presentation of the memory array. The memory display was presented for $753 \mathrm{msec}$ and was followed by a 1,000-msec blank period. A test array was then presented. On half of the trials, the test array exactly matched the memory array. On the other half of the trials, a single feature of a single object was changed to a value that had not been present in the memory array. The participant ceased repeating digits and verbally reported to an experimenter whether a change had occurred. The test array remained visible until this response was made.

The experiment was divided into three counterbalanced blocks. In the color block, the color of a single object was changed. In the shape block, the shape of a single object was changed. In the conjunction block, changes occurred (with equal probability) to either a single object's color or its shape, but not to both. The nature of the changes that would occur in each block was fully explained to each participant. Within each block, a 2 (change, no change) $\times 3$ (set size) $\times 14$ (repetition factor) design yielded 84 trials.

\section{Results}

Change detection performance was analyzed with a 2 (age group) $\times 3$ (set size) $\times 3$ (block) mixed model ANOVA (see Figure 1). Older adults exhibited poorer change detection performance $[F(1,22)=16.6, p<.001]$ and were more affected by set size $[F(2,44)=7.86, p<.001]$, but no other effects involving age were reliable (all $p s>.24$ ) and performance in all conditions was reliably greater than chance (all $p$ s <.01). For both groups - and to an equal extentperformance on the color block was more accurate and
Table 1

Mean Age (With Standard Deviations), Verbal IQ, and Formal Education of the Participants in Experiments 1, 2, and 3

\begin{tabular}{|c|c|c|c|c|}
\hline \multirow[b]{3}{*}{ Demographic } & \multicolumn{4}{|c|}{ Age Group } \\
\hline & \multicolumn{2}{|c|}{ Younger } & \multicolumn{2}{|c|}{ Older } \\
\hline & $M$ & $S D$ & $M$ & $S D$ \\
\hline \multicolumn{5}{|c|}{ Experiment 1} \\
\hline Age & 21.9 & 1.16 & 70.7 & 6.75 \\
\hline Verbal IQ & 111.7 & 3.75 & 109.3 & 3.85 \\
\hline Formal education & 16.6 & 1.08 & 14.4 & 3.09 \\
\hline \multicolumn{5}{|c|}{ Experiment 2} \\
\hline Age & 21.5 & 1.88 & 67.3 & 5.93 \\
\hline Verbal IQ & 110.7 & 4.81 & 110.2 & 4.90 \\
\hline Formal education & 16.8 & 2.40 & 16.6 & 3.40 \\
\hline \multicolumn{5}{|c|}{ Experiment 3} \\
\hline Age & 22.0 & 1.41 & 67.5 & 7.24 \\
\hline Verbal IQ & 109.7 & 4.88 & 106.2 & 3.85 \\
\hline Formal education & 16.9 & 0.793 & 14.6 & 3.13 \\
\hline
\end{tabular}

more resistant to set-size effects than that on the shape and conjunction blocks, ${ }^{1}$ which led to a reliable main effect of block $[F(2,44)=29.6, p<.001]$ and a reliable block $\times$ setsize interaction $[F(2,44)=7.86, p<.001]$. It is critical that analysis of the simple effects showed that performance in the conjunction block was often superior to - and never worse than-performance in the shape block. That is, the constraint on performance was the ability to retain information about shape, and not about bindings of shape and color. The results of Experiment 1 suggest that, although older adults could remember fewer objects than younger adults for both groups, VWM capacity was determined by the number of objects in the display, not the number of features that had to be remembered. The results of Experiment 1 therefore suggest that older adults store integrated object representations in VWM. In Experiment 2, we compared binding abilities of older and younger adults, using a procedure introduced by Wheeler and Treisman (2002) to correct for a potential limitation of the procedure used in Experiment 1.

\section{EXPERIMENT 2}

Because the changes across memory and test arrays in Experiment 1 involved only a single feature of a single item-even though the conjunction condition required participants to remember twice as many features as in the single feature blocks - performance could have been based on memory for the features that occurred in the display, without knowledge of which features occurred together. We corrected for this in Experiment 2 by replacing the conjunction condition with a binding condition, in which a pair of features were swapped between two items in the memory array (Wheeler \& Treisman, 2002). As in Experiment 1, performance in this binding condition was compared with that in the color and shape conditions, where changes were made by incorporating into the test array new features that had not been present in the memory array. However, since changes were made to two objects in the binding condition, in Experiment 2, the features of two objects were also changed in the color and shape conditions. If the object is the unit of VWM capacity, then performance in the binding 

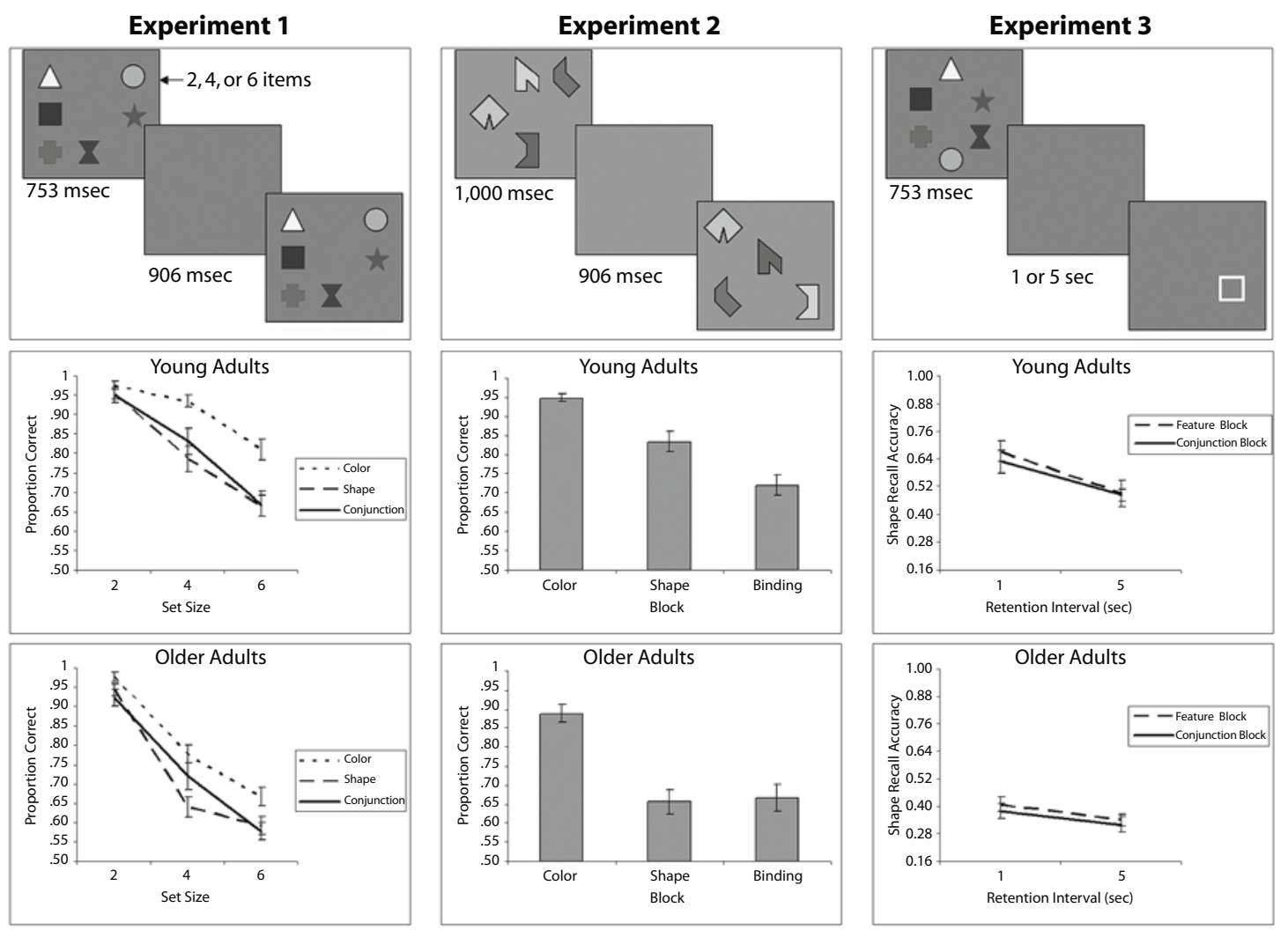

Figure 1. Top: Methods (stimuli were shown in color and are not drawn to scale). Middle: Results, younger adults. Bottom: Results, older adults. The $x$ - and $y$-axes cross at the chance level of performance. Error bars represent the standard error of the mean.

condition should be similar to that in the color and shape conditions. Conversely, if observers have difficulty binding features into integrated object representations, then memory for visual displays should be worse in the binding condition than in the color and shape conditions.

\section{Method}

Participants. A new sample of 12 healthy younger (mean age $=$ 21.5 years) and older (mean age $=67.3$ years) adults served as paid participants. The participant groups were matched on estimated verbal IQ and years of formal education (see Table 1).

Stimuli. Stimuli consisted of arrays of four objects placed randomly within an imaginary $3 \times 3$ square grid on a CRT monitor. Each object was defined by the combination of one of eight possible shapes and one of eight possible colors. To control for verbal rehearsal, novel stimuli were created that were visually discriminable, but for which it was difficult to assign a verbal or semantic label. On average, the objects subtended $.85 \mathrm{~cm}$ horizontally and vertically. The grid subtended $3 \mathrm{~cm}$ horizontally and vertically. Viewing distance was not constrained.

To formally assess the visual discriminability of the stimuli for each age group, we conducted a pilot experiment, in which older and younger observers simultaneously viewed two 4-object arrays. These arrays consisted of the same objects (arranged differently in each array), or they differed by two items. The arrays remained perceptually present until the participants had made a same-different judgment. To test the perception of shapes, each array consisted of four different shapes colored black. To test the perception of colors, each array consisted of the same shape presented in four different colors. No reliable age-group differences were observed (all $p \mathrm{~s}>.86$ ), with $A^{\prime}$ exceeding .93 in all conditions, indicating that the stimuli were highly discriminable for both groups and to an equal extent.

Design and Procedure. The experimental procedure is illustrated in Figure 1. On each trial, the memory array was presented for $1,000 \mathrm{msec}$, followed by a 900 -msec blank period. A test array was then presented. On half of the trials, the memory and test displays were composed of the same items. On the other half of the trials, the features of two objects were changed (see below). For both same and different trials, objects in the test array were presented in different locations from those in the memory array to ensure that the participant had to bind color and shape to each other, rather than to a location. As in Experiment 1, participants verbally reported to an experimenter whether a change occurred. The test array remained visible until this response was made.

The experiment was divided into three counterbalanced blocks. In the color block, arrays consisted of the same shape (randomly selected on each trial), presented in four different, randomly selected colors. In the shape block, arrays consisted of four different, randomly selected shapes presented in black. For both the color and the shape blocks, differences between memory and test arrays were made by changing the color or shape of two objects to randomly selected values that had not been present in the original memory array. In the binding block, arrays consisted of four different shapes presented in four different colors. In this block, changes were made between memory and test arrays by swapping colors between two objects. Within each block, a 2 (change, no change) $\times 16$ (repetition factor) design yielded 32 trials.

\section{Results}

Change detection performance was analyzed with a 2 (age group) $\times 3$ (block) mixed model ANOVA (see Figure 1). As in Experiment 1, older adults exhibited poorer 
change detection performance than that of younger adults $[F(2,22)=15.8, p<.001]$, indicating that older adults could remember fewer objects than younger adults. Additionally, a reliable main effect of block was observed $[F(1,22)=43.4, p<.001]$. These factors also interacted $[F(1,22)=3.83, p<.05]$. Planned comparisons demonstrated that for the young adults, performance on the color block exceeded that on the shape block $(p<.001)$, and performance on both single-feature blocks exceeded that on the binding block (all $p$ s $<.001$ ), revealing some degree of binding difficulty (see Wheeler \& Treisman, 2002, for a thorough discussion). For older adults, performance on the color block also exceeded that on the shape block $(p<.001)$; however, performance on the shape and binding blocks did not reliably differ $(p=.80)$. As in Experiment 1 , for older adults, the constraint on memory performance was the ability to retain information about shape, and not about bindings of shape and color. Consistent with the results of Experiment 1, these data suggest that older adults store integrated object representations in VWM. In Experiment 3, we turn our attention to binding abilities in recall tests.

\section{EXPERIMENT 3}

In Experiment 3, binding abilities were assessed with a recall task, using location as a cue. Recall tasks place greater demands on memory than those associated with recognition tasks; and because they impose greater selfinitiated processing demands, they generally reveal greater age-related loss of memory ability (see Craik, 2006). It is possible, therefore, that although age-related binding deficits were not observed using a recognition task in Experiments 1 and 2, deficits may occur when processing demands are increased in a recall task.

We followed a procedure introduced by Gajewski and Brockmole (2006): After participants had viewed an array of six colored shapes, one object was probed. In the singlefeature block, observers recalled the probed object's shape. In the conjunction block, observers recalled the probed object's shape and color. Thus, in the conjunction block, the number of task-relevant features was double that in the single-feature block. The dependent measure of interest was the accuracy of shape recall. If the object is the unit of capacity in VWM, then shape should be recalled with equal accuracy in both blocks. However, if observers have difficulty creating or maintaining bound object representations, then shape recall should be less accurate in the conjunction task. In addition to the change to a recall task, we also considered the impact of retention interval duration on memory. By manipulating the retention interval, we could determine whether any binding difficulties arise when objects must be maintained for longer periods of time in VWM.

\footnotetext{
Method

Participants. A new sample of 6 younger (mean age $=22.0$ years) and 12 older (mean age $=67.5$ years) healthy adults served as paid participants. The mean age of the sample was 67.5 years $(S D=$ 7.24). Although, on average, the younger adults had slightly more education, the groups were matched in terms of estimated verbal IQ (see Table 1).
}

Stimuli. Stimuli consisted of arrays of six objects positioned every $60^{\circ}$ around an imaginary circle with a diameter of $10 \mathrm{~cm}$. All other aspects of the stimuli were the same as in Experiment 1.

Design and Procedure. The experimental procedure is illustrated in Figure 1. First, to suppress verbal rehearsal, each trial began with the presentation of two digits that the observer vocally repeated throughout the trial. The participant pressed a button to terminate the digits' display, which was then replaced with a fixation cross centered on a gray background. A second buttonpress initiated the presentation of the memory array. The memory display was presented for $753 \mathrm{msec}$ and was followed by either a 1,000- or 5,000-msec blank period. A gray square frame appeared around one of the six locations in which an object was presented. In the single-feature block, the participant verbally reported the probed item's shape. In the conjunction block, he or she reported both the object's shape and its color. Within each block, a 6 (probe location) $\times 10$ (repetition factor) design yielded 60 trials.

\section{Results}

Recall performance was analyzed with a 2 (age group) $\times$ 2 (block) mixed model ANOVA. Figure 1 illustrates the results, broken down by these factors. Older adults exhibited poorer recall performance than that of younger adults $[F(1,16)=34.6, p<.001]$. Performance was also less accurate following a delay of 5,000 msec $[F(1,16)=$ $17.8, p<.001]$, a decrement that was marginally more pronounced for the younger adults $[F(1,16)=3.43, p=$ .08]. It is critical that no other interactions involving age were reliable (all $p \mathrm{~s}>.70$ ) — nor were any effects involving task (all $p \mathrm{~s}>$.29). ${ }^{2}$ As in Experiments 1 and 2, results indicate that although older adults could remember fewer objects than could younger adults, they showed no binding difficulties at either retention interval.

\section{GENERAL DISCUSSION}

This research considered how memory for visual objects, operationally defined as coherent sets of visual features (e.g., color and shape), are maintained in memory as we age. Previous research has demonstrated that in tasks involving longterm memory, older adults have more difficulty remembering combinations of features, even when memory for single features is preserved (Chalfonte \& Johnson, 1996). The present experiments are the first to investigate the possibility of analogous age-related binding deficits in VWM.

Due to the complexity of visual objects and the myriad features that define and discriminate them, an efficient memory system should combine multiple features into a singular object representation. Past research examining normal university-age participants has indeed suggested that objects are stored as integrated, singular, bound units in VWM, rather than as caches of individual features (Allen et al., 2006; Gajewski \& Brockmole, 2006; Johnson et al., 2008; Luck \& Vogel, 1997; Vogel et al., 2001). The results of the present experiments indicate that the same holds true for older adults. In both recognition and recall tasks, older adults could remember fewer objects than their younger counterparts, but they did not have difficulty creating bound representations of multifeature objects. Thus, these experiments found no evidence that differential memory performance in younger and older adults is linked to qualitatively different binding and storage mechanisms. 
At least two important contrasts can be drawn between the findings of this study and those of previous work, and these contrasts advance our theoretical understanding of binding processes in memory. First, although older adults may have difficulty remembering associations between objects and their locations in VWM (Cowan et al., 2006; Mitchell et al., 2000), binding deficits appear not to be a general characteristic of age-related memory difficulties. This finding, therefore, imposes limits on the generalizability of the associative deficit hypothesis (Naveh-Benjamin, 2000) as a complete explanation for age-related decline in episodic memory. Why age-related binding deficits in VWM exist for locations but not for features is an interesting question that lies beyond the scope of this study, but it is a question of both theoretical and practical importance. Given that few studies have considered binding mechanisms in VWM across the life span, it is possible that this variability is related to differences in experimental methodologies or in participant populations. Therefore, systematic and controlled studies of differences in feature and location binding across the life span are now required. Second, in contrast with tests of long-term memory, on which older adults do have difficulty remembering feature conjunctions (Chalfonte \& Johnson, 1996), no analogous pattern was found in VWM, suggesting that such deficits may be specific to the long-term memory storage system. This raises an interesting question for future research: Why do bindings dissociate following their transfer from VWM to visual long-term memory?

Although the present results suggest that older adults do not have a general difficulty creating bound representations of multifeature objects, they do not exclude the possibility that, under certain conditions, older adults are limited in their ability to create or maintain bindings. Prior research (see Bialystok \& Craik, 2006, for a review) has suggested that modality-general, age-related memory decline may be due to mounting deficits in mental processing speed (e.g., Salthouse, 1996), attention resources (e.g., Craik, 1986), and cognitive control (e.g., Zacks \& Hasher, 1994). It remains to be seen whether manipulations of these factors will differentiate binding abilities between older and younger adults.

\section{AUTHOR NOTE}

This research was supported by British Academy Grant SG-43564 awarded to J.R.B. and R.H.L; M.A.P. was further supported by Programme Alban, Scholarship No. E04D048179CO. We thank Michael Jin and Andrew McCullough for their help collecting data and three anonymous reviewers for helpful comments on a previous version of this article. Correspondence concerning this article should be addressed to J. R. Brockmole, Psychology Department, University of Edinburgh, 7 George Square, Edinburgh EH8 9JZ, Scotland (e-mail: james.brockmole@ed.ac.uk).

\section{REFERENCES}

Allen, R. J., Baddeley, A. D., \& Hitch, G. J. (2006). Is the binding of visual features in working memory resource-demanding? Journal of Experimental Psychology: General, 135, 298-313.

Bialystok, E., \& CRAIK, F. I. M. (EDS.) (2006). Lifespan cognition: Mechanisms of change. New York: Oxford University Press.

Chalfonte, B. L., \& Johnson, M. K. (1996). Feature memory and binding in young and older adults. Memory \& Cognition, 24, 403-416.

Cowan, N., Naveh-Benjamin, M., Kilb, A., \& Saults, J. S. (2006).
Life-span development of visual working memory: When is feature binding difficult? Developmental Psychology, 42, 1089-1102.

CRAIK, F. I. M. (1986). A functional account of age differences in memory. In F. Klix \& H. Hagendorf (Eds.), Human memory and cognitive capabilities: Mechanisms and performances (pp. 409-422). Amsterdam: Elsevier.

CRAIK, F. I. M. (2006). Remembering items and their contexts: Effects of aging and divided attention. In H. D. Zimmer, A. Mecklinger, \& U. Lindenberger (Eds.), Handbook of binding and memory: Perspectives from cognitive neuroscience (pp. 315-338). Oxford: Oxford University Press.

Gajewski, D. A., \& Brockmole, J. R. (2006). Feature bindings endure without attention: Evidence from an explicit recall task. Psychonomic Bulletin \& Review, 13, 581-587.

Johnson, J. S., Hollingworth, A., \& LuCK, S. J. (2008). The role of attention in the maintenance of feature bindings in visual short-term memory. Journal of Experimental Psychology: Human Perception \& Performance, 34, 41-55.

LuCK, S. J., \& Vogel, E. K. (1997). The capacity of visual working memory for features and conjunctions. Nature, 390, 279-281.

Mitchell, K. J., Johnson, M. K., Raye, C. L., Mather, M., \& D'Esposito, M. (2000). Aging and reflective processes of working memory: Binding and test load deficits. Psychology \& Aging, 15, 527-541.

Naveh-Benjamin, M. (2000). Adult age differences in memory performance: Tests of an associative deficit hypothesis. Journal of Experimental Psychology: Learning, Memory, \& Cognition, 26, 1170-1187.

Naveh-Benjamin, M., Guez, J., Kilb, A., \& Reedy, S. (2004). The associative memory deficit of older adults: Further support using facename associations. Psychology \& Aging, 19, 541-546.

Reuter-Lorenz, P. A., \& SyLVESTER, C.-Y. C. (2005). The cognitive neuroscience of working memory and aging. In R. Cabeza, L. Nyberg, \& D. Park (Eds.), Cognitive neuroscience of aging: Linking cognitive and cerebral aging (pp. 186-217). New York: Oxford University Press.

Salthouse, T. A. (1996). The processing-speed theory of adult age differences in cognition. Psychological Review, 103, 403-428.

Vogel, E. K., Woodman, G. F., \& LUCK, S. J. (2001). Storage of features, conjunctions, and objects in visual working memory. Journal of Experimental Psychology: Human Perception \& Performance, 27, 92-114.

WheELER, M. E., \& Treisman, A. M. (2002). Binding in short-term visual memory. Journal of Experimental Psychology: General, 131, 48-64.

ZACKS, R. T., \& HASHER, L. (1994). Directed ignoring: Inhibitory regulation of working memory. In D. Dagenbach \& T. H. Carr (Eds.), Inhibitory processes in attention, memory, and language (pp. 241-264). New York: Academic Press.

\section{NOTES}

1. This contrasts with Luck and Vogel (1997). However, Luck and Vogel tested memory for color and object orientation rather than shape. Among studies examining the binding of color and shape, including all three experiments presented here, more accurate memory for color is typically observed (e.g., Allen et al., 2006; Gajewski \& Brockmole, 2006; Johnson et al., 2008; Wheeler \& Treisman, 2002).

2. The lack of task effects could have arisen if observers ignored color information in the conjunction task. However, the accuracy of color recall always exceeded that for shape recall. For older adults, color was correctly recalled on $44 \%$ and $36 \%$ of trials following retention intervals of 1 and $5 \mathrm{sec}$, respectively (cf. shape memory of 38\% and 32\%). For younger adults, color recall was $78 \%$ and $59 \%$ accurate following retention intervals of 1 and $5 \mathrm{sec}$, respectively (cf. shape memory of $63 \%$ and $48 \%$ ). An ANOVA comparing age-related differences in color and shape memory within the conjunction block showed that younger adults exhibited overall better memory $[F(1,16)=40.6, p<.001]$, and color tended to be recalled better than shape $[F(1,16)=14.7, p<.001]$. However, the interaction was not reliable $[F(1,16)=3.21, p=.10]$, indicating that the age-related difference in memory for features in the conjunction block was not different for color and shape.

(Manuscript received September 4, 2007; revision accepted for publication January 7, 2008.) 